\title{
Venous thromboembolism in SARS-CoV-2 patients: only a problem in ventilated ICU patients, or is there more to it?
}

\author{
To the Editors:
}

Venous thromboembolism (VTE) is a well-known complication in hospitalised patients [1-5]. Risk factors include older age, obesity, immobilisation, active malignancy, systemic inflammatory response syndrome (SIRS), (major) surgery, thrombophilia and a history of thromboembolism [2, 5]. In 1884, Rudolph Virchow first described the underlying pathophysiological mechanisms, which consist of endothelial cell dysfunction/ inflammation, low blood flow and blood hypercoagulability. Current guidelines recommend the use of thromboprophylaxis in acutely ill medical patients who are at high risk for VTE (Padua score $\geqslant 4$, IMPROVE (International Medical Prevention Registry on Venous Thromboembolism) score $\geqslant 2$ ) [6]. However, in medical practice, less than half of the patients at risk receive adequate thromboprophylaxis [4].

In light of the current worldwide severe acute respiratory syndrome coronavirus 2 (SARS-CoV-2) outbreak, medical wards are confronted with increased numbers of acute severely ill patients. It is estimated that one in five SARS-CoV-2-infected patients require hospitalisation with a median stay of 11 days [7]. Together with older age ( $>65$ years) and a higher sequential organ failure assessment (SOFA) score $(>4)$, elevated D-dimer levels $\left(>1 \mu \mathrm{g} \cdot \mathrm{mL}^{-1}\right)$ are associated with in-hospital death [7]. D-dimer levels are a well-known but nonspecific biomarker of VTE, and they could indicate hypercoagulability. Elevated D-dimer levels also indicate inflammation. D-dimer levels are elevated in many other conditions beyond VTE, and its specificity for VTE diagnosis is low [7, 8]. Among hospitalised SARS-CoV-2 patients, $68 \%$ had D-dimer levels above the upper limit of normal, suggesting hypercoagulability in these patients [7]. Up to now, there is no clear association between SARS-CoV-2 infection and the presence of VTE, with the exception of a few case reports $[9,10]$. Nevertheless, it seems that these patients are at increased risk of VTE. First, elevated levels of pro-inflammatory cytokines are found in patients infected with SARS-CoV-2, leading to a highly inflammatory state in these patients [7]. Secondly, similar to the previous SARS coronavirus epidemic, angiotensin receptor 2 has been identified as a receptor that SARS-CoV-2 uses for cellular entry [11]. This receptor is highly expressed on the membrane of endothelial cells, and this could lead to endothelial cell-specific inflammation/dysfunction due to viral replication in these cells [12].

We assessed all patients hospitalised in the intensive care unit (ICU) and non-ICU ward of our centre on 3 April 2020. At that time in Belgium, 7297 patients were hospitalised due to SARS-CoV-2; the province Limburg had the highest incidence, 2607 infected patients, which corresponds with three infected patients per 1000 inhabitants. Patients in a palliative phase and/or who were non-contributive, mostly due to agitation or impaired mental status, were excluded. Insidious VTE was investigated using Doppler ultrasonography of the upper and lower limbs. ICU patients received $2 \times 40 \mathrm{mg}$ enoxaparin daily. Patients $>100 \mathrm{~kg}$ received $2 \times 60 \mathrm{mg}$ enoxaparin. In the non-ICU population, patients received $1 \times 40 \mathrm{mg}$ enoxaparin daily. Patients $>100 \mathrm{~kg}$ received $1 \times 60 \mathrm{mg}$ enoxaparin. None of the patients were on unfractionated heparin. For the statistical analysis, where appropriate, the Mann-Whitney or Chi-squared test was used for the analysis of patient characteristics using GraphPad Prism version 5.0.

@ERSpublications

Insidious venous thromboembolism (VTE) is mainly a problem in ICU-ventilated SARS-CoV-2 patients, while patients in the general ward, treated with thromboprophylaxis $\left(0.5 \mathrm{mg} \cdot \mathrm{kg}^{-1}\right)$, had a low incidence of insidious VTE https://bit.ly/2Yl8jft

Cite this article as: Criel M, Falter M, Jaeken J, et al. Venous thromboembolism in SARS-CoV-2 patients: only a problem in ventilated ICU patients, or is there more to it? Eur Respir J 2020; 56: 2001201 [https:// doi.org/10.1183/13993003.01201-2020]. 
In total, 82 patients were screened, 52 in the general ward and 30 in ICU. In ICU, 21 out of 30 patients were ventilated, none were on extracorporeal membrane oxygenation (ECMO); the other nine patients received nasal high flow oxygen. In total, six (7.3\%) patients had an insidious VTE (figure 1).

In the ICU, four patients had insidious VTE; all of these patients were ventilated. Among the 52 patients from the general ward, two had an insidious VTE, of which one had a risk factor (active stage IV cancer). If we consider the 49 patients (out of the 52) who benefit from prophylaxis, only one (2\%) of them developed VTE, compared with the incidence of VTE, four (13\%) out of 30, in ICU ward. None of the patients who accidentally did not receive thromboprophylaxis $(n=2)$, developed a VTE.

The patient demographics were comparable between the ICU and non-ICU (table 1). In both groups, being overweight seemed to be a clinically relevant issue in hospitalisation for SARS-CoV-2. Patients in the ICU had lower renal clearance at admission. No difference in D-dimer levels and cardiac enzymes was observed between both groups. Hypertension and diabetes were more prevalent in the cohort, without a difference between both groups. Up to 22 April 2020, mortality in the ICU group was four (13\%) out of 30 patients and in the non-ICU ward it was five (9.6\%) out of 52.

The current observation confirms a possible problem of thromboembolic events in SARS-CoV-2 critically ill ventilated patients. The insidious VTE seems mainly to be a problem in ICU ventilated patients, while patients on the general ward, treated with thromboprophylaxis, had a low incidence of insidious VTE. In our cohort, two patients had insidious VTE in the non-ICU population, and both had an apparent predisposing risk factor. One other patient had a tiny clot in the left internal jugular vein. This is also described in other viral diseases, such as influenza A [13], although in this case, without any finding of thrombosis in either of the lower limbs. Cultures were negative, no sign of sepsis. Control after 3 days was negative for VTE.

VTE with adequate thromboprophylaxis is prevalent in $5-15 \%$ of critically ill patients in the ICU [14]. In this cohort, we identified $13 \%$, which is in line with previous data. Thromboprophylaxis failure $(n=5)$ in our cohort (in the ICU and non-ICU) was 6.1\%, comparable with landmark studies MEDENOX (MEDical Patients with ENOXaparin trial) [15], for non-critically ill patients, and PROTECT (Prophylaxis for Thromboembolism in Critical Care trial) [16], for critically ill patients where thromboprophylaxis failure of $5-6 \%$ is reported. In the ventilated SARS-CoV-2 population, four (19\%) out of 21 developed VTE. Until now, no clear correlation with the underlying disease has been found. These results were somewhat lower than previously published studies.

In the study by CUI et al. [17], the authors reported VTE in $25 \%$ of the patients, who did not receive thromboprophylaxis. KLOK et al. [18] reported that 27\% developed VTE, with inclusion of pulmonary embolism. We should remark that the incidence of VTE in our study is perhaps underestimated, as we only looked at VTE in the lower and upper limbs. For practical reasons and owing to disease severity, no computed tomography scan was performed in patients. In addition, pulmonary embolism was not included in this study.

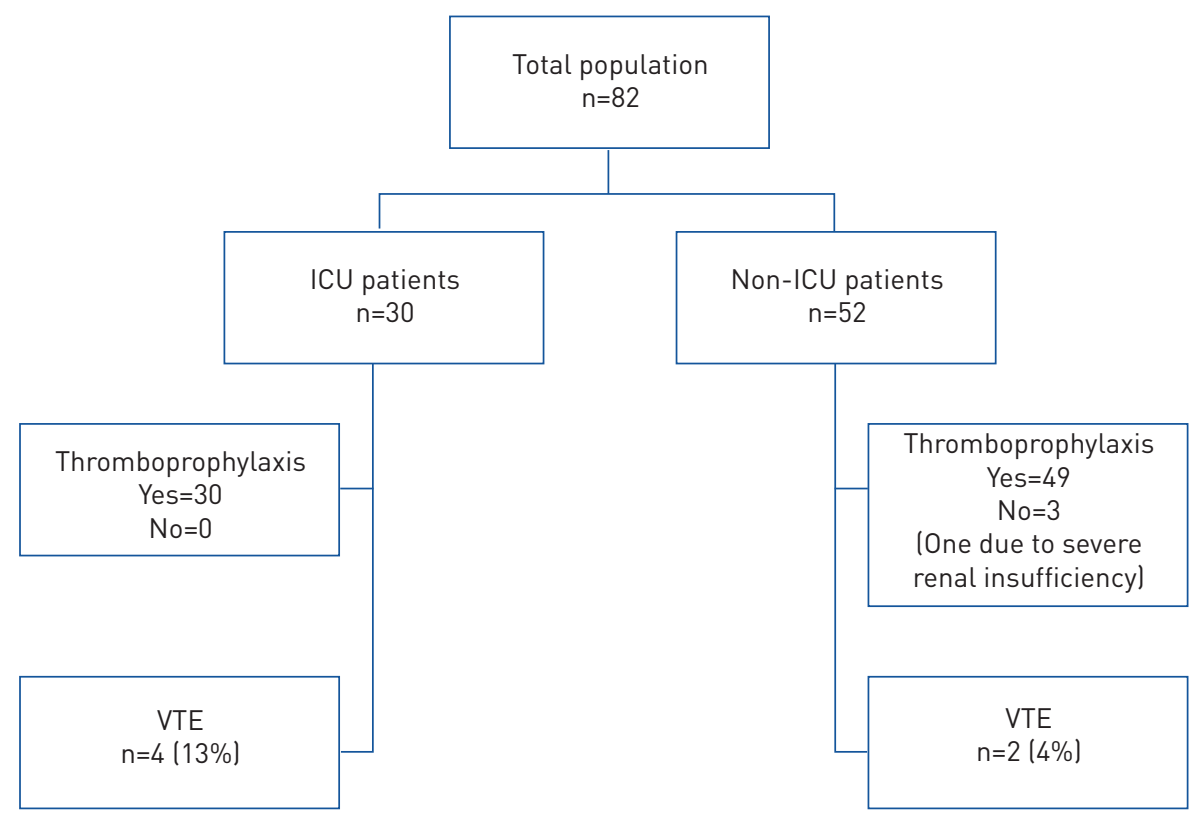

FIGURE 1 Patient population. ICU: intensive care unit; VTE: venous thromboembolism. 
TABLE 1 Patient demographics

\begin{tabular}{lccc} 
& Non-ICU & ICU & p-value \\
\hline Subjects $\mathbf{n}$ & 52 & 30 & \\
Deep venous thrombosis & $2(4)$ & $4(13)$ & 0.18 \\
Age yrs & $63.6 \pm 14.4$ & $64.5 \pm 11.8$ & 0.86 \\
Males & $28(54)$ & $20(67)$ & 0.35 \\
BMI & $29.9 \pm 5.8$ & $30.3 \pm 5.4$ & 0.72 \\
Renal function EGFR $\mathbf{~} \mathbf{m} \cdot \mathbf{m i n}^{-1} \cdot \mathbf{1 . 7 3} \mathbf{~ m}^{-\mathbf{2}}$ & $77 \pm 20$ & $64 \pm 20$ & 0.0037 \\
D-dimer at presentation $\boldsymbol{\mu} \mathbf{g} \mathbf{f i b . e q .} \cdot \mathbf{~ m L}^{-1}$ & $1.2 \pm 1.1$ & $1.4 \pm 1.0$ & 0.18 \\
Troponines at presentation $\mathbf{n g} \cdot \mathbf{L}^{-1}$ & $19.9 \pm 24.7$ & $20.7 \pm 23.2$ & 0.84 \\
History of arterial hypertension & $20(38)$ & $10(33)$ & 0.64 \\
History of diabetes & $9(17)$ & $5(17)$ & 0.94
\end{tabular}

Data are presented as $\mathrm{n}(\%)$ or mean $\pm \mathrm{SD}$, unless otherwise stated. ICU: intensive care unit; $\mathrm{BMI}$ : body mass index; EGFR: estimated glomerular filtration rate.

D-dimer levels at presentation seem to be no clear marker of the development of insidious VTE or outcome in our cohort, perhaps due to the evaluated levels that we detected in our whole SARS CoV-2 population. The VTE cohort $(n=6)$ is too small to perform adequate analysis and draw a conclusion on the patients with VTE and the predictive value of D-dimer levels. Further, as previously described in other cohorts, patients who were overweight, and had hypertension and diabetes were at risk of hospitalisation due to SARS-CoV-2 [19].

In conclusion, we believe that our single-centre study may provide relevant preliminary data on the hot topic of thromboprophylaxis in the SARS-CoV-2 population. Further exploration and follow-up data are needed to confirm SARS-CoV-2 as a risk factor of thromboembolic events.

Maarten Criel $\odot^{1}$, Maarten Falter $\oplus^{1}$, Jasmien Jaeken ${ }^{1}$, Margaretha Van Kerrebroeck ${ }^{1}$, Isabelle Lefere $^{2}$, Liesbeth Meylaerts ${ }^{2}$, Dieter Mesotten ${ }^{3,4}$, Margot vander Laenen, Tom Fivez ${ }^{4}$, Michiel Thomeer $\oplus^{1,3}$ and David Ruttens ${ }^{1}$

${ }^{1}$ Dept Pulmonary Medicine, Ziekenhuis Oost Limburg, Genk, Belgium. ${ }^{2}$ Dept Radiology, Ziekenhuis Oost Limburg, Genk, Belgium. ${ }^{3}$ Dept of Medicine and Life Sciences, Hasselt University, Diepenbeek, Belgium. ${ }^{4}$ Dept of Anaesthesiology, Intensive Care, Emergency Medicine and Pain Therapy, Ziekenhuis Oost-Limburg, Genk, Belgium.

Correspondence: David Ruttens, Ziekenhuis Oost-Limburg, Pulmonary Medicine, Schiepse Bos 6, Genk 3600, Belgium. E-mail: david.ruttens@zol.be

Received: 15 April 2020 | Accepted after revision: 28 April 2020

This article has editorial commentaries: https://doi.org/10.1183/13993003.01608-2020 and https://doi.org/10.1183/ 13993003.01634-2020

Conflict of interest: None declared.

\section{References}

1 Spyropoulos AC, Anderson FA, FitzGerald G, et al. Predictive and associative models to identify hospitalized medical patients at risk for VTE. Chest 2011; 140: 706-714.

2 Anderson FA Jr, Spencer FA. Risk factors for venous thromboembolism. Circulation 2003; 107: 91-16.

3 Tapson VF, Decousus H, Pini M, et al. Venous thromboembolism prophylaxis in acutely ill hospitalized medical patients. Chest 2007; 132: 936-945.

4 Cohen AT, Tapson VF, Bergmann J-F, et al. Venous thromboembolism risk and prophylaxis in the acute hospital care setting (ENDORSE study): a multinational cross-sectional study. Lancet 2008; 371: 387-394.

5 Kaplan D, Casper TC, Elliot CG, et al. VTE incidence and risk factors in patients with severe sepsis and septic shock. Chest 2015; 148: 1224-1230.

6 Schünemann HJ, Cushman M, Burnett AE, et al. American Society of Hematology 2018 guidelines for management of venous thromboembolism: prophylaxis for hospitalized and nonhospitalized medical patients. Blood Adv 2018; 2: 3198-3225.

7 Zhou F, Yu T, Du R, et al. Clinical course and risk factors for mortality of adult inpatients with COVID-19 in Wuhan, China: a retrospective cohort study. Lancet 2020; 395: 1054-1062.

8 Pabinger I, Ay C. Biomarkers and venous thromboembolism. Arterioscler Thromb Vasc Biol 2009; 29: 332-336.

9 Danzi GB, Loffi M, Galeazzi G, et al. Acute pulmonary embolism and COVID-19 pneumonia: a random association? Eur Heart J 2020; 41: 1858.

10 Xie Y, Wang X, Yang P, et al. COVID-19 complicated by acute pulmonary embolism. Radiol Cardiothorac Imaging 2020; 2: e200067-2. 
11 Hoffmann M, Kleine-Weber H, Krüger N, et al. The novel coronavirus 2019 (2019-nCoV) uses the SARScoronavirus receptor ACE2 and the cellular protease TMPRSS2 for entry into target cells. BioRxiv 2020; preprint [https://doi.org/10.1101/2020.01.31.929042].

12 Hamming I, Timens W, Bulthuis M, et al. Tissue distribution of ACE2 protein, the functional receptor for SARS coronavirus. A first step in understanding SARS pathogenesis. J Pathol 2004; 203: 631-637.

13 Porquet-Bordes V, Guillet E, Cammas B, et al. Lemierre syndrome and influenza A (H1N1). Arch Pediatr 2011; 18: 413-415.

14 Boddi M, Peris A. Deep vein thrombosis in intensive care. Adv Exp Med Biol 2017; 906: 167-181.

15 Samama MM, Cohen AT, Darmon JY, et al. A comparison of enoxaparin with placebo for the prevention of venous thromboembolism in acutely ill medical patients. Prophylaxis in Medical Patients with Enoxaparin Study Group. N Engl J Med 1999; 341: 793-800.

16 PROTECT Investigators for the Canadian Critical Care Trials Group and the Australian and New Zealand Intensive Care Society Clinical Trials Group, Cook D, Meade M, et al. Dalteparin versus unfractionated heparin in critically ill patients. N Engl J Med 2011; 364: 1305-1314.

17 Cui S, Chen S, Li X, et al. Prevalence of venous thromboembolism in patients with severe novel coronavirus pneumonia. J Thromb Haemost 2020; in press [https://10.1111/jth.14830].

18 Klok FA, Kruip MJHA, van der Meer NJM, et al. Incidence of thrombotic complications in critically ill ICU patients with COVID-19. Thromb Res 2020; in press [https://10.1016/j.thromres.2020.04.013].

19 Muniyappa R, Gubbi S. COVID-19 pandemic, corona viruses, and diabetes mellitus. Am J Physiol Endocrinol Metab 2020; 318: E736-E741. 